Enfance en difficulté

\title{
Les contributions de la pédagogie comportementale à l'éducation spécialisée
}

\section{Jacques Forget}

Volume 1, mars 2012

URI : https://id.erudit.org/iderudit/1012122ar

DOI : https://doi.org/10.7202/1012122ar

Aller au sommaire du numéro

\section{Éditeur(s)}

Université Laurentienne

ISSN

1920-6275 (imprimé)

1929-8544 (numérique)

Découvrir la revue

Citer cet article

Forget, J. (2012). Les contributions de la pédagogie comportementale à l'éducation spécialisée. Enfance en difficulté, 1, 7-45.

https://doi.org/10.7202/1012122ar

\section{Résumé de l'article}

La pédagogie comportementale est apparue au début du $20^{\mathrm{e}}$ siècle avec les travaux d'Edward Lee Thorndike, Frederick Lister Burk et Sidney Pressey. Il faudra cependant attendre l'ouvrage de B.F. Skinner, The technology of teaching, publié en 1968, pour que cette conception de l'enseignement trouve une place appréciable parmi les modèles pédagogiques disponibles dans les écoles. Combiné à l'émergence de nombreuses techniques d'analyse de tâche, d'orientation comportementale ou cognitive, l'enseignement programmé et ses variantes se sont développés de manière importante au cours des années 60 et 70. La technologie de l'instruction est alors devenue un domaine de recherche et une orientation pédagogique légitimes. Dix ans plus tard, l'arrivée d'une conception cognitive " globaliste » de l'apprentissage a rapidement dominé la pensée didactique. Quinze ans plus tard, cette orientation s'est transformée en didactique socioconstructivisme, la version pédagogique du postmodernisme qui se situe aux antipodes de la tradition empirique et du doute rationaliste en attribuant, dans sa version radicale, comme celle de von Glasersfeld (2009), la même valeur épistémologique à toutes les formes de connaissances, qu'elles soient scientifiques, intuitives ou mythiques. L'approche soutient aussi que toutes les compétences d'un enfant, qu'elles soient disciplinaires ou transversales, ne peuvent provenir que de sa propre construction subjective. Mais au-delà de ces transformations, l'enseignement programmé a masqué l'apport de nombreux travaux de recherche qui furent développés dans le contexte de l'éducation spécialisée et de projets d'intégration scolaire et qui donnèrent lieu au développement de modèles et de méthodes d'enseignement s'appuyant sur la psychologie comportementale. Le présent article vise à faire un survol de ces principaux modèles. À la fin de ce panorama, quelques expériences réalisées au Canada français au cours des 50 dernières années sont brièvement présentées. 
www.laurentienne.ca

\section{Les contributions de la pédagogie comportementale à l'éducation spécialisée}

\section{Jacques Forget \\ Université du Québec à Montréal}

La pédagogie comportementale, terme relativement inusité dans les pays francophones, s'est développée de manière substantielle au cours des années 60 et 70 grâce aux travaux de chercheurs en psychologie et en éducation comme Becker (1977), Bereiter et Engelmann (1966), Bloom (1968), Carnine et Silbert (1979), Engelmann (1969, 1980), Gagné (1965), Gilbert (1962), Homme (1970), Keller (1968), Mechner (1967), Mager (1962), Pennypacker, Koenig et Lindsley (1972) et Skinner (1968). Il faut aussi mentionner les réalisations de quelques psychologues et pédagogues russes comme Schestakow (1968) ou Talysina (1980) qui avaient cependant été précédées des travaux importants de Galperine (1980). En France, il faut souligner les travaux relativement négligés de Couffignal (1963) sur la pédagogie cybernétique. Il est vrai que sur le plan strictement historique, les toutes premières propositions furent présentées au début du $20^{\mathrm{e}}$ siècle par Thorndike (1912), Burk (1908) et Pressey (1926). Et il est possible d'ajouter à cette liste les travaux de Dewey (1916) dans la mesure où son pragmatisme social et éthique constituait, avec le fonctionnalisme individuel de Thorndike, une conception sœur de la psychologie béhavioriste qui émergeait alors des propositions programmatiques de Watson $(1914,1919)$.

La pédagogie comportementale constitue un domaine particulier de la psychologie comportementale. Celle-ci est issue du béhaviorisme, de l'analyse du comportement et de quelques travaux en psychologie cognitive comme ceux d'Anderson (1968), Anderson, Reder 
et Simon (1996), Merrill (1978) ou Scandura (1966, 1973). Nous utilisons dans ce texte les termes de psychologie comportementale et pédagogie comportementale et non celui de béhaviorisme parce que ce dernier n'a pas seulement influencé la psychologie ou l'éducation, mais aussi bien d'autres disciplines comme la criminologie (Akers, 1973), la sociologie (Burgess, \& Bushell, 1969), la microéconomie (Kagel, Battalio, \& Green, 1995), l'anthropologie (Harris, 1979), les sciences politiques (Ulman, 1979), la psychiatrie (Wolpe, 1958), la médecine (Fontaine, Kulbertus, \& Etienne, 1996), le travail social (Gambrill \& Stein, 1978), les sciences vétérinaires cliniques (Overall, 1997) et l'entraînement d'animaux domestiques (Pryor, 1984).

Dans le domaine des sciences de l'éducation, la psychologie comportementale s'est développée dans deux directions. La première a proposé une série de stratégies d'intervention permettant à l'enseignant d'améliorer la gestion de sa classe, particulièrement auprès des enfants qui présentent des difficultés d'apprentissage ou d'adaptation psychosociale. Ce domaine est tantôt désigné par l'expression d'analyse et de modification du comportement, tantôt par celle d'analyse appliquée du comportement. Les problèmes abordés sont multiples : troubles du comportement, troubles des conduites, problèmes d'attention à la tâche, trouble déficitaire de l'attention avec ou sans hyperactivité, troubles d'apprentissage, troubles de la communication et du langage comme le mutisme électif, troubles du spectre autistique. Les stratégies sont multiples : façonnement, enchaînement progressif ou à rebours, modelage, renforcement social différentiel, renforcement différentiel d'autres comportements, renforcement différentiel de comportements incompatibles, techniques opérantes avec jetons incluant des contingences individuelles ou de groupe, contrats de contingence, autocontrôle (p. ex., auto-évaluation, autonotation, autorenforcement, autodétermination des standards de performance), guidance adaptée, contrôle du stimulus, estompage, loi de l'appariement, et quelques autres (Alberto \& Troutman, 2003; Cooper, Heron, \& Heward, 1987; Martens \& Meller, 1990; Ninness, McCuller, \& Ozenne, 2000; Vargas, 2009; Wolery, Bailey, \& Sugai, 1988). Dans la presse populaire comme dans un nombre incalculable de textes d'orientation constructiviste ou socioconstructiviste (voir p. ex., Archambault, 1994), ce domaine est généralement réduit à l'utilisation de « systèmes de jetons » ou de "systèmes d'émulation " qui s'appuieraient sur le renforcement extrinsèque et qui auraient l'immense défaut de miner la motivation intrinsèque des élèves (pour une réponse détaillée à cette critique, voir Cameron \& Pierce, 2002) ou encore 
sur la répétition et la pratique intensive (Mayer, 1996) que plusieurs appellent l'exercisation (Tardif, 1998). En fait, l'un des problèmes est que les concepts et les principes fondamentaux à partir desquels sont construites ces stratégies ne sont plus enseignés (et les plus récentes ne l'ont jamais été) dans les départements d'éducation québécois et trop d'auteurs comme Gauthier (1994) ont une vision tronquée d'un champ d'expertise professionnel comme l'analyse appliquée du comportement, croyant qu'elle se ramène à l'utilisation de récompenses, si ce n'est de punitions.

La seconde direction est celle qui touche la pédagogie et la didactique. Là aussi, la vision projetée est souvent parcellaire. Ainsi, en sciences de l'éducation ou en psychologie de l'éducation, l'enseignement programmé représenterait le modèle exemplaire de la pédagogie comportementale. Si l'affirmation fut relativement exacte au cours des années 60 , plusieurs chercheurs utilisent toujours l'affirmation pour démontrer que cette conception de l'enseignement est morte, justifiant ainsi les notices nécrologiques qui témoigneraient de la disparition de la pédagogie comportementale (Boulet, 1999). Pourtant, même si l'intérêt pour l'enseignement programmé en tant que pratique pédagogique spécifique a diminué de manière substantielle (Vargas \& Vargas, 1992), plusieurs de ses éléments sont intégrés à l'intérieur des nouvelles technologies de l'information (Desrochers \& Gentry, 2004; Ingvarsson \& Hanley, 2006; Marquis \& Lavoie, 1998; Merrill, 1996). Cela dit, il est vrai que sa popularité a masqué la présence d'autres modalités d'enseignement comportementalistes comme l'enseignement direct, l'enseignement de précision, Comprehensive Application of Behavior Analysis to Schooling $\left(C A B A S^{\circledR}\right)$, Morningside model of generative instruction, l'enseignement explicite, l'enseignement systématique ou l'enseignement personnalisé.

L'objectif de cet article est de présenter un tour d'horizon de ces modèles pédagogiques qui ont eu une certaine influence en éducation spécialisée. De manière plus concrète, l'article inclut trois sections portant sur autant de thématiques :

(a) la conceptualisation théorique de l'apprentissage et de l'enseignement défendue par la pédagogie comportementale;

(b) la présentation des éléments essentiels de cinq de ces modèles qui, faut-il le préciser, ont surtout été mis en place dans les pays anglo-saxons;

(c) quelques projets et expériences provenant de praticiens ou de chercheurs d'origine canadienne-française. 
Évidemment, dans le contexte d'un article, il est impossible de brosser un tableau complet de ces différentes contributions. Même si la pédagogie comportementale n'a jamais eu une très grande popularité et quoi qu'en disent des auteurs comme Boulet (1999), cette orientation n'a jamais constitué un paradigme (voir Forget, 2009) - les milliers d'études publiées depuis les cinquante dernières années sont difficiles à répertorier. En outre, un certain nombre d'expériences francophones n'ont pas toujours fait l'objet de publications dans des revues savantes et le matériel a eu une diffusion généralement très limitée. Dans certains cas, nous n'avons pas été en mesure de le retrouver. Enfin, il est possible que certaines études en milieu francophone, au Québec ou ailleurs au Canada français, nous aient échappées. Nous nous en excusons auprès des auteurs.

\section{Les fondements théoriques de la pédagogie comportementale}

La pédagogie comportementale s'intéresse depuis toujours aussi bien à l'apprentissage qu'à l'enseignement. Au cours des années 90, un certain nombre d'auteurs d'orientation cognitive tels Tardif et Presseau (1998) ou Boulet (1999) ont proposé l'idée selon laquelle l'enseignement traditionnel correspondrait à un paradigme (terme qui provient de la thèse de Thomas Kuhn sur la structure des révolutions scientifiques) appelé le «paradigme de l'enseignement», et l'enseignement contextualisé d'orientation cognitive correspondrait à un autre paradigme, le «paradigme de l'apprentissage». Nonobstant le fait que ces auteurs défigurent totalement le concept de paradigme (pour Kuhn, il ne peut y avoir deux ou trois paradigmes qui se côtoient dans le temps; et lorsqu'ils se suivent, il existe toujours une période anarchique entre la disparition de l'ancien et l'émergence du nouveau), la thèse n'a jamais été appuyée par une quelconque étude empirique ou historique qui pourrait démontrer hors de tout doute raisonnable l'incompatibilité des deux «paradigmes ». Ensuite, cette dichotomie enseignement versus apprentissage est ancrée dans un contexte linguistique et culturel limité. Par exemple, dans la littérature scientifique germanophone, les deux termes sont rarement dichotomisés, même chez les chercheurs d'orientation cognitiviste (De Corte, Geerligs, Peters, Lagerweij, \& Vandenberghe, 1996). Autrement dit, les auteurs francophones qui tentent de démontrer l'incommensurabilité des deux concepts oublient qu'au-delà de leur interprétation très personnelle de la thèse de Kuhn 
(1972), la distinction n'est aucunement universelle. Et le plus paradoxal est que ces mêmes auteurs associent la psychologie béhavioriste au paradigme de l'enseignement plutôt qu'à celui de l'apprentissage, oubliant sans doute que le béhaviorisme s'est essentiellement intéressé aux processus d'apprentissage et aux stratégies concrètes qui favorisent l'apparition de ses diverses manifestations tant sur le plan instrumental et attitudinal-motivationnel que sur le plan verbal, cognitif et social (Leduc, 1989; Staats, 1986). D'ailleurs, les psychologues du développement et les éthologistes lui ont souvent reproché, à tort ou à raison, cette amitié exclusive.

La pédagogie comportementale s'appuie sur neuf hypothèses :

(a) tous les enfants, quels que soient leurs particularités, leurs difficultés, leur déficience ou leur handicap, peuvent apprendre;

(b) la majorité des enfants peuvent apprendre le contenu des programmes scolaires ordinaires et développer les compétences cognitives, verbales, instrumentales, affectives et sociales qui en découlent, s'ils bénéficient d'un enseignement systématique ou d'un entraînement adéquat - en fait, l'élève apprend ce qu'on lui enseigne et il apprend rarement ce qu'on ne lui enseigne pas;

(c) l'intégration des enfants en difficulté en classe ordinaire est préférable à la classe spéciale ou à l'école spéciale, pourvu que les ressources matérielles et humaines nécessaires à leur maintien dans ce milieu soient intégrées en classe ordinaire;

(d) l'enseignement de n'importe quel contenu d'apprentissage exige une analyse de trois éléments qui constituent ce qu'il est convenu d'appeler l'équation comportementale S-R-C (Giroux, 1979) : les règles et les stimuli discriminatifs présentés par l'enseignement (S), l'analyse de tâche permettant de déterminer et de hiérarchiser le contenu d'apprentissage, généralement, mais pas toujours, du simple au complexe (R), les contingences de renforcement qui soutiennent le comportement, les acquisitions et les progrès de l'enfant $(\mathrm{C})$;

(e) l'enfant doit démontrer une très bonne maîtrise d'un contenu d'apprentissage avant d'être exposé à un contenu plus complexe;

(f) l'évaluation formelle et empirique del'efficacitéd'un enseignement constitue une composante non négociable :la recherche de données probantes de nature empirique et la promotion des meilleures pratiques sont au cœur de la pédagogie comportementale; 
(g) l'évaluation de l'apprentissage et des progrès de l'élève doit s'appuyer sur une analyse quantitative, directe, atomisée, "critériée », normative et régulière plutôt que sur une évaluation qualitative, subjective, indirecte, globale et occasionnelle;

(h) le transfert des apprentissages doit faire l'objet d'une planification pédagogique formelle et structurée, plutôt que d'être uniquement souhaitée;

(i) le premier facteur qui permet d'évaluer l'efficacité d'un modèle d'enseignement est la qualité des apprentissages de l'enfant et le rythme de sa progression.

Notons qu'il existe plusieurs ouvrages de langue anglaise consacrés à la pédagogie comportementale (Gardner et al., 1994; Moran \& Malott, 2004; Ninness et al., 2000; West \& Hamerlynck, 1992) quoique la plupart portent sur un modèle particulier. En outre, il existe depuis 1991 une revue consacrée à ce domaine, le Journal of Behavioral Education. En revanche, en français, la moisson est chétive. D’une part, il n'existe aucun ouvrage consacré au domaine. Et les quelques rares revues qui publiaient, à l'occasion, des études empiriques, comme La technologie du comportement ou la Revue de modification du comportement, sont disparues. La Revue des sciences de l'éducation publiait aussi quelques études associées à l'orientation (p. ex., celle de Cadieux et Boudreault, 2003), mais curieusement le terme de pédagogie comportementale (ou de béhaviorisme) n'était jamais utilisé.

\section{Les principaux modèles en pédagogie comportementale}

Si tous les modèles comportementalistes de l'enseignement partagent l'ensemble de ces caractéristiques, leurs différences s'expliquent par la modélisation particulière qu'ils en font et par l'importance relative accordée à chacune d'entre elles.

\section{L'enseignement direct}

L'enseignement direct est une méthode d'enseignement proposée dès 1964 à l'Université de Chicago par Siegfried Engelmann et Carl Bereiter (Bereiter \& Engelmann, 1966). Par la suite vont se greffer des chercheurs associés directement à l'analyse appliquée du comportement comme Wesley Becker (1977) et Douglas Carnine (Carnine et al., 1997) et d'autres associés aux sciences de l'éducation comme Barak Rosenshine et Robert Stevens (Rosenshine \& Stevens, 1986). 
Les premières réalisations tangibles sur le plan pédagogique furent l'édition d'une série de programmes d'enseignement de la lecture et des mathématiques au primaire appelé DISTAR pour Direct Instruction System for Teaching Arithmetic and Reading (l'acronyme signifie aussi Direct Instruction Systems for Teaching and Remediation). Notons qu'au début des années 80 , quelques éléments de ce matériel furent traduits et adaptés au Québec ( $\mathrm{La}$ Petite École) et offerts aux enseignants comme matériel individualisé et autocorrecteur d'enrichissement. Le matériel se présentait sous la forme de petites fiches plastifiées et de couleurs différentes en fonction du degré de difficulté de la tâche.

L'enseignement direct s'articule autour de deux volets composés réciproquement de six éléments pédagogiques et de sept stratégies didactiques. Nous utilisons ici le terme de didactique en tant qu'actions de l'enseignant lui permettant de présenter un contenu d'apprentissage dans le but «de faire réaliser par les élèves des objectifs déterminés » (De Corte, Geerligs, Peters, Lagerweij, \& Vandenberghe, 1996, p. 145), tout en étant conscients que les distinctions entre pédagogie, didactique et didaxologie ne font aucunement l'unanimité parmi les chercheurs en sciences de l'éducation.

Sur le plan pédagogique, l'enseignement direct inclut sept composantes :

(a) construire les programmes d'enseignement (p. ex., en lecture, en écriture, en sciences, en mathématiques) de manière très structurée;

(b) décomposer les éléments du programme en plusieurs sousobjectifs opérationnels et concrets exempts de toute ambiguïté (il s'agit ici d'utiliser les techniques d'analyse de tâches les plus validées sur le plan empirique ou rationnel);

(c) maximiser la quantité d'informations en fonction du temps alloué aux acquisitions scolaires;

(d) évaluer les habiletés déjà acquises par chacun des élèves;

(e) fournir un guide pédagogique qui présente les tâches attendues et les explications nécessaires à l'enseignement de chaque notion ou de l'algorithme de résolution du problème — l'enseignant doit minimiser l'improvisation;

(f) préparer plusieurs évaluations formatives et normatives permettant d'évaluer rapidement et fréquemment le progrès des élèves; 
(g) reconnaître, en tant qu'enseignant, ses propres limites et son ignorance devant certaines questions des élèves.

Sur le plan didactique, l'enseignement direct privilégie sept stratégies particulières :

(a) présenter clairement la règle (p. ex., l'accord du pluriel), la notion (p. ex., la caractéristique des légumes) ou l'algorithme (p. ex., faire une multiplication avec deux décimales) en évitant de poser des questions pièges, des questions triviales ou des questions floues;

(b) présenter des exemples simples qui découlent directement de la règle;

(c) présenter en alternance des exemples qui répondent à la règle et des contre-exemples - ici l'enseignement direct s'inspire du système Ruleg (rule and e.g.; c'est-à-dire règle et exemple) proposé en 1962 par Evans, Homme et Glaser;

(d) exiger des élèves des réponses claires et complètes;

(e) inviter les élèves à compléter une série d'exercices qui découlent directement de l'objectif d'apprentissage et à répéter l'activité jusqu'à ce que leur performance soit automatisée et fluide;

(f) utiliser des procédures de renforcement positif suite aux succès des élèves;

(g) enseigner aux élèves la technique d'auto-instruction (angl. covertization) qui consiste à se dire intérieurement la règle ou l'algorithme de résolution de problèmes.

Notons qu'il existe deux versions de l'enseignement direct. La première est celle de Bereiter et Engelmann (1966), Adams et Engelmann (1996) ou encore Carnine et al. (1997). Elle insiste non seulement sur les composantes déjà décrites, mais propose une série de curriculums précis dans la plupart des matières scolaires du primaire et du secondaire. Les auteurs utilisent les majuscules pour la désigner (angl. Direct Instruction ou DI). Cette situation explique pourquoi il est difficile d'appliquer l'Enseignement Direct en milieu non anglophone puisqu'il n'existe pratiquement aucun document pédagogique construit à partir des principes didactiques proposés par ses artisans. La deuxième version, décrite par Rosenshine et Stevens (1986), inclut une série de modèles pédagogiques qui respectent les différentes étapes de l'Enseignement Direct tout en utilisant d'autres types de curriculums. Les auteurs utilisent les minuscules (angl. direct instruction) pour désigner ces modèles. 
L'enseignement direct est devenu relativement connu lors de l'implantation du programme américain Follow Through à la fin des années 60 par le Bureau de l'éducation des États-Unis suite aux initiatives du président de l'époque, Lynden B. Johnson. Cette initiative faisait suite à l'implantation d'un autre projet national, Head Start, programme éducatif qui visait les enfants d'âge préscolaire. À l'époque, l'objectif du gouvernement était de combattre la pauvreté sur l'ensemble du territoire américain. Et ce combat passait obligatoirement par une meilleure scolarisation des enfants, particulièrement des enfants en difficulté.

En fait, devant l'échec de la scolarisation des élèves provenant de milieux défavorisés, le Bureau de l'éducation proposa aux chercheurs d'évaluer de manière formelle et empirique l'efficacité des modèles qu'ils jugeaient efficaces, particulièrement dans le cas de ceux qui présentaient des difficultés. Deux conditions générales étaient exigées des équipes de recherche pour obtenir un financement gouvernemental :

(a) le modèle devait viser l'apprentissage d'habiletés scolaires de base (lire, écrire, compter), l'apprentissage d'habiletés cognitives complexes (p. ex., le transfert) et l'apprentissage d'habiletés socio-émotives (p. ex., l'estime de soi);

(b) le modèle devait être appliqué en milieu socioéconomique modeste incluant au moins une école expérimentale et une école témoin, cette dernière devant favoriser un enseignement traditionnel.

Watkins (1997) rappelle que 22 équipes de recherche proposèrent leur modèle (Tucson early education, Cognitively oriented curriculum, Direct instruction, Individualized early learning program, Language development approach, Mathamagenic activities program, Responsive education program, Bank street, Behavior analysis, Florida parent education, Interdependent learning, Open education, Hampton Institute nongraded, Culturally linguistic, Parent supported application of the behavior oriented prescriptive teaching approach, Home-school partnership model and motivational approach, Role trade, New school approach, Culturally democratic learning environments, Parent implementation approach, California process, Responsive environments early childhood model). De ces propositions, 13 équipes furent en mesure d'implanter leur modèle suivant les exigences du Bureau de l'éducation. Notons que, de toute l'histoire de l'éducation au $\mathrm{XX}^{\mathrm{e}}$ siècle, ce projet fut le plus ambitieux tant sur le plan des ressources financières et humaines qui lui furent consacrées que sur le plan de la qualité scientifique exigée. 
$\mathrm{Au}$ terme de trois ans d'application auprès de plusieurs milliers d'élèves du primaire, seul l'enseignement direct put démontrer son efficacité sur le plan des apprentissages scolaires de base, sur le plan des habiletés cognitives complexes et sur le plan de l'estime de soi des élèves. Le modèle d'enseignement s'appuyant sur l'utilisation des contingences de renforcement (behavior analysis model) s'est avéré plus efficace que l'enseignement traditionnel sur le plan des apprentissages scolaires de base, particulièrement en mathématiques, un peu plus efficace sur le plan de l'estime de soi, mais moins efficace sur le plan des habiletés cognitives (Rhine, 1981). Tous les autres modèles n'ont pu démontrer de résultats supérieurs à ceux obtenus par les écoles témoins. Qui plus est, la plupart d'entre eux se sont avérés «moins » efficaces que l'enseignement traditionnel (Bissonnette, Richard, \& Gauthier, 2006; Stebbins, St-Pierre, Proper, Anderson \& Cerva, 1977; Watkins, 1997). Pourtant, en 1980, le Bureau de l'éducation mit fin au financement des projets d'enseignement direct sous prétexte qu'il faillait donner la chance aux autres modèles de démontrer leur intérêt ou leur efficacité. Cependant, certains projets purent se poursuivre jusqu'en 1995 (Bissonnette, Richard, \& Gauthier, 2006). Comme le soulignent Watkins (1997) et Péladeau et Legault (2000), cette décision et le silence subséquent des établissements d'enseignements supérieurs ainsi que celui de nombreux chercheurs représentent le plus important scandale $\mathrm{du} \mathrm{XX}^{\mathrm{e}}$ siècle dans le domaine de l'éducation.

Notons qu'il existe une association américaine qui s'intéresse à la promotion de l'enseignement direct, l'Association for Direct Instruction (ADI). Depuis 2001, l'association publie un bulletin de nouvelles, Direct Instruction News et une revue scientifique, le Journal of Direct Instruction. Le congrès annuel de l'ADI rassemble près de 800 congressistes provenant principalement des États-Unis et du Canada.

\section{L'enseignement personnalisé}

L'enseignement personnalisé (angl. personalized instruction) a été développé par Fred Keller, l'un des pionniers, avec Skinner, de l'analyse du comportement. Ainsi, au début des années 60, l'Université de Brasilia demande à Keller de participer à l'organisation pédagogique $\mathrm{du}$ nouveau département de psychologie. Celui-ci propose alors un modèle d'enseignement où les cours magistraux ne deviennent plus la principale source d'information pour les étudiants. En 1968, il publie dans le Journal of Applied Behavior Analysis un article au titre élo- 
quent, "Good-Bye Teacher... ». D’une certaine manière, et en dépit de l'existence du programme au Brésil, ce texte va devenir le point de départ du développement de l'enseignement personnalisé, non seulement dans certains programmes universitaires américains, mais aussi dans quelques écoles primaires et secondaires. Comme bien d'autres modèles d'enseignement d'orientation comportementale, l'enseignement personnalisé ne cherche pas à corriger les difficultés de certains élèves, mais à les prévenir en assurant un certain degré d'individualité au processus d'enseignement.

L’enseignement personnalisé présente cinq caractéristiques qui touchent le rythme d'apprentissage des élèves, le degré de maîtrise d'une habileté, l'enseignement individualisé, l'enseignement collectif et l'utilisation d'autres étudiants en tant que tuteurs (angl. proctor). Ainsi, ce modèle, comme les autres, reconnaît que les personnes apprennent à des rythmes différents compte tenu de leurs antécédents et de leurs caractéristiques personnelles. Il faut donc que le système accepte que certains d'entre eux puissent réussir un objectif d'apprentissage avant ou après les autres.

Cela dit, il est impératif que chaque contenu d'apprentissage soit très bien maîtrisé avant que l'élève soit exposé à un nouveau contenu. Dans le système de Keller, ce degré de maîtrise est d'au moins $90 \%$. Évidemment, l'une des conditions pour qu'une telle individualisation puisse se réaliser est de diviser le contenu de l'apprentissage en unités bien hiérarchisées et de le rendre disponible à tous les élèves sous forme écrite - le support peut être un document papier en encore un document électronique. Autrement dit, tout le contenu d'un cours est découpé en un certain nombre de modules d'apprentissage. Pour organiser ce contenu, il faut en faire une analyse de contenu. Notons que l'enseignement personnalité ne propose pas une technique particulière d'analyse de contenu. L'enseignant ou l'établissement qui décide d'adopter une telle démarche peut adapter n'importe quelle procédure d'analyse de tâche comme l'analyse comportementale de Mechner (1967) qui s'appuie sur l'hypothèse selon laquelle l'apprentissage d'un concept ou la résolution d'un problème implique trois habiletés : la discrimination, la généralisation et le transfert; le système Ruleg de Evans et al. (1962) ou encore l'analyse contingente de Gropper (1974). L'enseignant peut évidemment s'appuyer sur son expérience pour identifier l'ordre optimal de présentation du contenu. La disponibilité du contenu sous forme papier ou sous forme électronique est une 
condition nécessaire dans la mesure où le cours magistral, au sens classique du mot, n'existe plus. Lélève acquiert les connaissances au moment où il le désire, en respectant évidemment les échéanciers du programme. Pour aider l'étudiant dans son étude, un certain nombre de tuteurs sont disponibles. L'apprentissage de chaque module est suivi d'une évaluation formelle gérée par le tuteur. Lorsque l'élève obtient un score de $90 \%$ ou plus, il peut avoir accès au module suivant. Le cours magistral avec l'enseignant n'est disponible qu'au moment où un certain nombre d'élèves ont réussi un ou plusieurs modules. Ce cours ne sert donc plus à donner des informations sur les principes de base d'un contenu. L'étudiant n'est plus assujetti à prendre des notes ou à faire répéter l'enseignant parce qu'il n'a pas compris. En revanche, ce type d'apprentissage permet à l'enseignant d'exposer des applications afin de favoriser le transfert des connaissances. Le cours devient aussi un lieu d'échange entre les étudiants et l'enseignant. Le contexte et les discussions deviennent donc une source de motivation dans la mesure où les étudiants ne cherchent plus à se faire expliquer les notions de base, mais bien à échanger sur les implications et les applications des concepts acquis.

Notons que le rôle des tuteurs est de quatre ordres. Ils assurent la passation et la correction des mini-tests en offrant plusieurs plages horaires aux étudiants. Ils enseignent les notions aux étudiants lorsque ceux-ci ne parviennent pas à maîtriser le contenu suite à la lecture des documents et à l'accomplissement des nombreux exercices pratiques que le matériel offre. Ils doivent offrir l'aide technique qui pourrait faciliter l'apprentissage. Par exemple, les technopédagogues qui sont embauchés par certaines universités, certains collèges ou certaines écoles secondaires, pourraient jouer ce rôle. Enfin, les tuteurs doivent être une source de facilitation sociale, de stimulation et d'encouragement pour les élèves.

Même si le modèle a d'abord été conçu dans le cadre de la formation universitaire, plusieurs écoles secondaires et plusieurs collèges américains ont adopté l'enseignement personnalisé. Ainsi, en 1977, plus de 1200 établissements d'enseignement américains avaient adopté le modèle de Keller. En 1988, Pear et Kinsner ont développé la première version informatisée d'un contenu d'enseignement (un cours universitaire sur les techniques d'analyse et de modification du comportement) basé sur le modèle de Keller. Le programme, appelé CAPSI / PC, devenait donc disponible en tout temps aux étudiants. Son contenu fut planifié en fonction de la hiérarchie des apprentissages de Bloom. 
En milieu francophone, l'une des rares expériences a été réalisée par Magerotte dans le cadre de son enseignement au département d'orthopédagogie de l'Université de Mons-Hainaut en Belgique (Magerotte \& Willaye, 2010).

Sur le plan de l'efficacité, le modèle a été comparé à plusieurs autres modèles pédagogiques. Ainsi, dans une méta-analyse regroupant plus de 300 études, Kulik, Kulik et Cohen (1979) ont montré que l'enseignement personnalisé est plus efficace que l'enseignement traditionnel, que l'enseignement assisté par ordinateur, que l'enseignement programmé ou que l'enseignement par audio-tutorat.

\section{L'enseignement de précision}

L'enseignement de précision (angl. precision teaching) a été développé au cours des années 60 par Ogden Lindsley et Henry Pennypacker. Contrairement aux autres modèles présentés dans cet article, l'enseignement de précision n'est pas à proprement parler un modèle d'enseignement, mais bien une procédure particulière, plus ou moins complexe, d'évaluation du progrès d'une personne placée dans une situation d'apprentissage, d'entraînement ou de travail. Ce modèle consiste essentiellement à inscrire sur un graphique semi-logarithmique appelé graphique standard de l'enseignement de précision, la performance quotidienne de l'élève. Les bornes de l'ordonnée permettent d'inscrire des comportements dont le débit peut varier entre 1000 comportements à la minute et 1 comportement par 1000 minutes. L'abscisse correspond à un déroulement temporel quotidien. Autrement dit, chaque ligne verticale du graphique correspond à une journée spécifique de la semaine : dimanche, lundi, mardi, etc.

Le graphique permet aussi d'inscrire une série de renseignements pertinents à l'analyse des données d'observation sur la performance de l'élève : performance quotidienne des réussites et des erreurs, plancher de notation, plancher de comportement, plafond de notation, objectif terminal, accélération minimale attendue du progrès, accélération obtenue, amélioration globale.

Notons que depuis quelques années, des versions informatisées du graphique existent. Le plus récent et sans aucun doute le plus performant est celui mis au point par la société québécoise Cocon : le Graphique Standard modifié Automatisé, version 24.2 (http://cocon.ca/ et http://www.clinique-autisme-asperger-mtl.ca/NormandGiroux.html). 
La fonction de ce logiciel est d'informatiser le processus d'enseignement de précision et de l'analyse appliquée du comportement.

En 1980, Patrick McGreevy fonde le Journal of Precision Teaching, une revue dont le principal objectif est de publier les graphiques de différents projets, que ces derniers soient réalisés en contexte scolaire, en contexte de réadaptation ou en contexte de travail. En 1990, un groupe de chercheurs fonde la Standard Celeration Society afin de coordonner de manière intégrée les différentes activités professionnelles et scientifiques des utilisateurs de l'enseignement de précision, particulièrement les conférences annuelles et la revue qui devient le Journal of Precision Teaching and Celeration.

Dans la mesure où l'enseignement de précision s'appuie sur une vision radicalement inductive de la recherche et dans la mesure où elle représente essentiellement un instrument de mesure, il n'existe pas de véritable étude expérimentale visant à évaluer son efficacité par rapport à d'autres traditions de mesure et d'évaluation en pédagogie. Son efficacité est simplement illustrée par les milliers d'études publiées depuis les années 60. D'ailleurs, dès 1971, une banque de données appelée Behavior Bank avait été développée par Lindsley et Koening (voir Forget, 2009). En 1974, 3362 projets d'enseignement de précision avaient été répertoriés. Les résultats montrent que les projets qui utilisent des techniques d'intervention comportementale associées au contrôle du stimulus (les antécédents dans l'équation $S-R-C$ ) sont plus efficaces que les stratégies qui s'appuient sur le renforcement positif, ce qui correspond, d'une certaine façon, aux fondements de l'enseignement direct.

\section{$\mathrm{CABAS}^{\circledR}$}

Le Comprehensive Application of Behavior Analysis to Schooling $\left(C A B A S^{\circledR}\right)$ a été développé au début des années 80 par R. Douglas Greer comme modèle pédagogique basé sur les principes de l'analyse appliquée du comportement pour enseigner la musique à des jeunes élèves. Par la suite, le modèle fut utilisé pour enseigner les matières scolaires de base auprès d'enfants en difficulté. Le projet de Greer (1994; voir aussi Greer \& Keohane, 2004) se voulait une réaction à l'affirmation de Brophy qui soutenait en 1983 qu'en dépit de leurs succès dans le domaine de la gestion des comportements difficiles en classe, les techniques d'analyse appliquée du comportement n'avaient pas eu véritablement d'impact en pédagogie, et qu'elles ne pourraient aucunement en avoir. Au cours des 
années 90 , le développement du modèle $C A B A S{ }^{\circledR}$ s'est poursuivi et son champ d'application s'est étendu à l'ensemble du curriculum de l'école primaire.

En fait, le modèle ne propose pas une manière particulière d'enseigner comme le fait l'enseignement direct. Il ne propose aucune stratégie didactique originale. Il vise plutôt à intégrer une série de traditions comportementalistes et de pratiques pédagogiques " instructionnistes " afin de proposer ce que Greer et Keohane (2004) appellent une véritable science pédagogique de la scolarisation. Cette science ne porte pas uniquement sur les stratégies d'enseignement, mais aussi sur le rôle des étudiants, des parents, des enseignants, de leurs superviseurs et des directions d'école. Le modèle intègre aussi l'entraînement aux comportements verbaux comme ceux de désignation (angl. tact training) et de demandes (angl. mand training), s'appuyant ainsi sur la théorie fonctionnaliste du comportement verbal de Skinner (1957) et sur l'entraînement systématique de répertoires verbaux (p. ex., désigner des choses, des concepts, des procédures), le tutorat de groupe, le système de communication PECS (Picture Exchange Communication System, proposé par Bondy et Frost, 1994), l'utilisation de scripts, l'enseignement personnalisé, l'enseignement programmé par ordinateur, l'évaluation de précision, la médiation par les pairs, l'entraînement à la fluidité (angl. fast rate training) qui provient de la tradition de l'enseignement de précision (cette tradition utilise plutôt l'expression fluidity building). Le modèle intègre aussi l'autonotation, les contrats de contingences, l'enseignement incident (renforcer naturellement, rapidement et fréquemment les apprentissages et les comportements appropriés amorcés par l'élève dans les contextes de la vie quotidienne) ainsi que plusieurs éléments de l'enseignement direct comme la présentation d'exemples positifs et d'exemples négatifs.

Le modèle $C A B A S^{\circledR}$ est surtout utilisé par des écoles américaines, anglaises et irlandaises. À notre connaissance, aucune expérience n'a été réalisée en milieu francophone. Le seul projet qui peut se rapprocher de celui-ci est Primes, que nous verrons plus loin. Évidemment, les raisons qui expliquent cette situation sont simples, connues et identiques à celles qui expliquent l'absence pratiquement totale de la pédagogie comportementale en milieu francophone.

\section{The Morningside model of generative instruction}

Le Morningside model of generative instruction développé par Kent Johnson au cours des années 80 représente l'un des modèles les plus 
intégrateurs des caractéristiques de la pédagogie comportementale. En effet, il combine plusieurs traditions béhavioristes en éducation : le pragmatisme éducatif de Dewey, l'analyse du comportement de Skinner, l'enseignement direct d'Engelmann, Carnine et Becker, l'enseignement de précision de Pennypacker, Koenig et Lindsley (1972), l'analyse de tâche de Markle et Tiemann (1967), la mesure de la fluidité comportementale de Binder (1993), la théorie de la créativité et de la générativité d'Epstein (1991) et, dans une moindre mesure, la théorie du développement et de l'enseignement découlant des travaux de Vygotsky (1986).

Le modèle s'adresse d'abord aux élèves qui présentent des retards pédagogiques et le programme se déroule à l'intérieur d'écoles spéciales appelées Morningside. Les élèves bénéficient du programme pendant les sessions d'été ou encore pendant deux ou même trois ans. Il faut noter que les écoles Morningside sont des établissements privés, les parents devant assumer une grande partie des coûts. En revanche, l'école garantit que le progrès de l'enfant doit correspondre au double du progrès moyen des enfants de l'école publique. Si les résultats ne sont pas atteints, les parents sont remboursés. Johnson et Street (2004) affirment qu'au cours de son histoire, l'école a dû remettre les frais de scolarité à moins de $1 \%$ des parents.

Le modèle vise l'acquisition des habiletés de base et d'habiletés complexes en lecture, en écriture et en mathématiques. À titre d'illustration, les habiletés associées à la lecture incluent la conscience phonologique, l'écoute et le langage expressif, le décodage, la construction du vocabulaire, les habiletés cognitives liées à la compréhension et les stratégies d'auto-évaluation. Notons que le modèle vise non seulement les habiletés de base dans les matières scolaires, mais aussi des compétences associées à la citoyenneté (ici l'influence de John Dewey est manifeste) et celles associées aux émotions.

Le modèle est offert aux élèves fréquentant l'école primaire ou l'école secondaire. En fait, il est surtout offert aux élèves qui présentent un trouble déficitaire de l'attention, un trouble d'hyperactivité ou des retards pédagogiques. Quelques études ont porté sur les enfants ayant un trouble du spectre de l'autisme ou une déficience intellectuelle légère.

Le modèle vise l'acquisition de six habiletés que l'on pourrait qualifier de transversales si l'expression n'était pas rattachée au socioconstructivisme : la rétention, l'endurance, l'application, la performance, la stabilité et la fluidité. 


\section{Quelques modèles développés au Canada français}

Dans cette section, nous présentons six modèles d'enseignement d'orientation comportementale qui ont été mis en place dans certaines écoles du Québec ou dans des écoles françaises de l'Ontario. Certaines de ces expériences ont été de courte durée, d'autres plus prolongées. Évidemment, la comparaison avec les études américaines est difficile, puisque le soutien financier de ces expériences par les organismes scolaires correspond rarement à ce qui prévaut aux États-Unis. En outre, la plupart d'entre eux ont été développés par des intervenants du milieu (enseignants, orthopédagogues, conseillers pédagogiques, psychologues) et non par des chercheurs universitaires. Il reste que les quelques mesures empiriques prises pour évaluer ces programmes dépassent souvent ce que les chercheurs qui défendent des modèles pédagogiques comme la pédagogie de la mise en situation, la pédagogie contextualisée, l'enseignement global de la lecture ou le socioconstructivisme ont présenté jusqu'ici. Lorsque nous parlons de mesures empiriques, il ne s'agit pas ici de questionnaires distribués aux enseignants pour mesurer leur degré de satisfaction face à telle ou telle méthode proposée ou dans le but de connaître leur évaluation subjective du progrès des élèves (ce qui ne constitue évidemment aucunement des mesures invalides ou inutiles), mais bien de recueillir des informations sur le rendement de l'enfant. Comme le soulignent Péladeau, Forget et Gagné (2010), il ne s'agit pas d'évaluer la valeur d'une réforme scolaire ou d'une méthode pédagogique par les discours qui ont contribué à sa mise en place ou ceux qui lui permettent de se maintenir, mais par ses effets concrets et pragmatiques sur la réussite de tous les élèves.

Nous avons exclu de cette courte recension les quelques modèles qui présentent certaines caractéristiques de la pédagogie comportementale et qui ont été mis en place dans le cadre de l'enseignement ordinaire, non pour intervenir auprès des enfants en difficulté ou à risque, mais pour mieux individualiser l'enseignement. Les deux plus importants furent sans doute Le Progrès continu et le Système d'apprentissage géré par l'étudiant (SAGE). Le Progrès continu fut proposé par le ministère de l'Éducation du Québec au début des années 70 et mis en place dans les écoles primaires de la Commission des écoles catholiques de Montréal, C.É.C.M. (aujourd'hui la Commission scolaire de Montréal). Le modèle s'appuyait en grande partie sur la pédagogie de la maîtrise de Bloom (1968). Les curriculums en français et en mathématiques du primaire 
étaient divisés en 24 étapes (quatre par année scolaire). Au terme de chaque étape, l'élève était convié à répondre à un test d'évaluation critérié. L'obtention d'une performance de $80 \%$ ou plus lui permettait de poursuivre. Lidée des concepteurs du programme était que le primaire pouvait être réalisé en six ans pour l'ensemble des élèves, en sept ans pour ceux en difficulté, et en cinq ans pour les plus doués (C.É.C.M., 1973; Forget, Otis, \& Leduc, 1988). Le second, SAGE, fut mis en place à la fin des années 70 par plusieurs chercheurs en éducation (voir Burton, 1978) et l'Institut national de recherche scientifique (INRS-éducation). Basé lui aussi sur la pédagogie de la maîtrise de Bloom et sur l'enseignement individualisé, le programme visait l'apprentissage des matières de base en français et en mathématiques. Il fut surtout mis en place dans des écoles de la Rive-Sud de Montréal. Contrairement au Progrès continu, le modèle fut comparé à l'enseignement collectif traditionnel dans près de 20 classes. Au début des années 80 , il fut surtout utilisé dans le cadre de l'enseignement de l'anglais langue seconde (Bégin, 1978; Bégin \& Dussault, 1980; Burton, 1978).

Le Progrès continu disparut parce qu'il entraînait nécessairement la disparition de la classe degré traditionnelle. En fait, le respect intégral du modèle aurait provoqué une véritable révolution éducative au Québec et l'émergence des écoles dites à aires ouvertes de l'époque aurait pu faciliter, sur le plan de l'aménagement physique, cette disparition. Dans le cas de $S A G E$, deux raisons expliquent sa disparition. D'une part, il faut rappeler la disparition, à cette époque, de l'INRS-éducation comme centre d'expertise québécoise en éducation et l'émergence très rapide de la pédagogie cognitiviste "globaliste " au début des années 80 qui fit tabula rasa des quelques tentatives d'enseignement individualisé qui s'appuyaient sur l'apprentissage par objectifs et sur des analyses de tâches ou d'expériences en pédagogie comportementale. Nous pouvons affirmer que, sauf exception, le travail de démolition était terminé au milieu des années 80 .

\section{MÉLI}

À la fin des années 70, un groupe de praticiens et de chercheurs associés à la Commission scolaire du Sault-St.-Louis en banlieue de Montréal et à la C.É.C.M. développent un programme d'enseignement de la lecture (Méthode expérimentale de lecture individualisée ou MÉLI) qui s'appuie sur la théorie de l'apprentissage social et du béhaviorisme paradigmatique d'Arthur Staats (1986). L'équipe de recherche est alors 
composée d'Annie Déjean, Christiane Grenier, Lise Journault, Jean Davidson et Christian Hottlet (voir Hottlet, 1981).

D'emblée, l'association de MÉLI à la théorie de Staats est assumée. Les auteurs affirment d'ailleurs que la méthode n'est pas adaptable et se veut une alternative aux trop nombreux « hochepots pédagogiques " qui sévissent souvent en éducation. En ce sens, la méthode partage avec l'enseignement direct l'idée selon laquelle l'enseignant n'a pas à inventer, chaque fois, un nouveau matériel au goût du jour.

Selon Staats (1986), l'apprentissage de la lecture requiert cinq niveaux d'habiletés, appelées ici des répertoires de personnalité :

(a) la discrimination des lettres (habileté grapho-phonétique);

(b) la reconnaissance des unités de lecture qui consiste à établir la correspondance entre les graphèmes et les phonèmes;

(c) le décodage;

(d) la lecture de mots entiers;

(e) la lecture de phrases.

En 1981, Déjean, Hottlet et Goulet publient cinq modules de lecture appelés MÉLI. Un premier rapport d'expérimentation (Hottlet \& Déjean, 1980) rapporte des résultats significatifs de la performance et des progrès des élèves de première et deuxième année de deux écoles situées en milieu défavorisé de Montréal : l'école Champlain et l'école St-Anselme. Lapplication du programme se poursuivit dans quelques autres écoles (voir p. ex., Journault, 1982), mais il n'est pas clair à quel moment précis ces applications prirent fin. En revanche, il est clair qu'au milieu des années 80, la plupart des conseillers pédagogiques du Québec à qui ce type de matériel était présenté le dénonçaient vertement suivant l'argument selon lequel l'enseignement de la lecture devait se faire par l'intermédiaire de méthodes globales (qui, soit dit en passant, n'ont jamais été en mesure de démontrer leur efficacité : voir Bissonnette et al., 2006).

\section{LIRE}

Le programme LIRE a été développé à la C.É.C.M. à la fin des années 70 par Réal Montambeault, Jean Archambault, Nicole Nadeau-Savoie et Georges Ouellet (1979). Ces auteurs considéraient que le matériel de $M E ́ L I$ était peu adapté à des élèves du primaire qui éprouvaient des difficultés en lecture, mais dont les acquisitions étaient telles que les derniers modules du programme MÉLI étaient, en partie, trop faciles. 
Sur le plan pédagogique, le matériel LIRE, constitué de quatre séries de fiches de lecture, est présenté de manière similaire à celui de $M E ́ L I$ en respectant une démarche du simple au complexe (Montambeault, Archambault, \& Ouellet, 1979). Le guide méthodologique propose aussi la mise sur pied d'un système de jetons contingents à la réussite des élèves. Ici aussi, le système s'appuie sur les principes de l'enseignement personnalisé qui prévoit que l'élève doit obtenir une performance d'au moins $90 \%$ à chaque étape pour avoir accès aux fiches subséquentes. Cependant, s'il est possible d'utiliser des tuteurs comme dans le cas de $M E ́ L I$, le programme s'adresse d'abord aux orthopédagogues qui rencontrent les élèves en difficulté dans un contexte de « dénombrement flottant » (l'orthopédagogue intervient auprès de l'élève dans un petit local à raison de trois ou quatre périodes par semaine).

\section{L'enseignement de précision}

$\mathrm{Au}$ Québec, l'enseignement de précision a connu des hauts et des bas, surtout des bas. Dès le milieu des années 70, le modèle a été défendu par Normand Giroux à la Faculté d'éducation de l'Université de Sherbrooke. D'ailleurs, il avait développé un cours de trois crédits qui fut offert aux étudiants du programme d'orthopédagogie. Lors de son départ de l'université en 1978, le cours fut assumé par le présent auteur. Cependant, la Faculté mit fin à l'aventure quatre ans plus tard. Au total, près de 300 étudiants ont bénéficié de cette formation. En revanche, peu d'entre eux l'ont appliquée dans leur pratique pédagogique. Les raisons de cette situation, analysées en détails par Giroux et Forget (1996), sont d'abord associées à la vague cognitiviste " globaliste » qui a déferlé sur le Québec à cette époque, sur l'absence d'une tradition évaluative quantitative et continue des apprentissages en contexte scolaire dans la plupart des écoles du Québec, sur l'ampleur de la tâche des orthopédagogues et sur la complexité inhérente de la technique.

Par la suite, quelques rares études ont utilisé la technique auprès d'enfants en difficulté. Les trois principales études, publiées dans le domaine scolaire, sont celles de Bélanger (1982), Quintin (1982) et Giroux et Forget (2000).

La première (Bélanger, 1982) a été réalisée auprès de 16 élèves de $4^{\mathrm{e}}$ année dont huit présentent un trouble du comportement et huit un trouble d'apprentissage. L'objectif est d'améliorer leur performance en mathématiques, leur comportement de coopération et leur attention à la tâche. Les élèves sont placés en équipes de deux. Chaque équipe a 
un seul graphique d'enseignement de précision et les exercices se font toujours en équipe. Trois fois par semaine, un court examen est complété de manière individuelle. Cependant, c'est la moyenne de l'équipe qui est inscrite sur le graphique et qui donne droit à des stimuli de renforcement. Lidée ici est que la contingence de groupe devrait inciter l'élève le plus performant à mieux aider son partenaire lors des exercices. Utilisant un protocole de recherche à niveaux de base multiples, l'auteure montre que la procédure permet une amélioration significative du rendement scolaire des élèves. En revanche, la fréquence de leurs comportements de coopération est relativement stable, suggérant que le programme d'entraînement de cinq heures fourni aux élèves n'est pas suffisant pour les amener à enseigner à leurs pairs et à les renforcer.

L'étude de Quintin (1982) vise à évaluer l'effet de l'auto-évaluation, de l'autonotation (à l'aide d'un graphique agrandi du graphique standard d'enseignement de précision) et d'un matériel de lecture silencieuse sur la performance en lecture de dix élèves de deuxième année qui reçoivent des services d'orthopédagogie. Les résultats montrent une amélioration de la performance, mais non une amélioration de la vitesse d'apprentissage. En outre, si l'autonotation a un certain effet sur cette performance, le fait de demander aux élèves de s'auto-évaluer ne modifie aucunement cette performance.

L'étude de Giroux et Forget (2000) vise, d'une part, à évaluer l'intérêt d'une version modifiée du graphique standard de l'enseignement de précision (l'empan est réduit de 200 comportements à la minute à 1 comportement à la minute, ce qui permet d'amplifier les écarts et ainsi faciliter la détection des moindres changements de la performance de l'élève) auprès de cinq orthopédagogues et, d'autre part, à évaluer l'impact de cet instrument de mesure sur la performance de 52 élèves en difficulté d'apprentissage sur leur rendement en lecture. Les résultats montrent que la version modifiée est préférée à la version classique, que l'utilisation du graphique permet une amélioration significative de la performance des élèves, mais surtout au début de programme.

\section{Primes}

Le terme d'enseignement systématique désigne un groupe plus ou moins homogène de modèles pédagogiques qui défendent l'importance de l'individualisation de l'enseignement et de l'évaluation récurrente des apprentissages des élèves. Dans un texte publié par le Conseil supérieur de l'Éducation (Allard et al., 1974), cette conception de 
l'enseignement proviendrait des travaux de R.W. Tyler sur le curriculum scolaire, des principes de la psychologie comportementale, des théories de l'apprentissage et de la technologie de l'instruction.

En 1977, Forget propose à la Commission scolaire MargueriteBourgeois un modèle d'enseignement appelé Primes (Programme de recherche sur l'implantation d'un modèle d'enseignement systématique) dont l'objectif est de favoriser le maintien des élèves qui présentent un trouble du comportement, un trouble d'apprentissage, un trouble du développement ou une déficience intellectuelle en classe ordinaire dans leur école de quartier (voir Forget, 1981). Le modèle est construit à partir de la pédagogie de la maîtrise, de l'enseignement personnalisé, de l'application du principe de Premack, des concepts d'apprentissage " par objectifs minima " (les contenus et les habiletés qui devraient être maîtrisés par tous les élèves), de la théorie de l'apprentissage de Staats, de l'enseignement de précision et des techniques d'analyse appliquée du comportement sur le plan de la gestion de la classe. Le programme se décompose en six objectifs correspondant à six niveaux possibles d'implication des enseignants :

(a) évaluer les effets d'une séquence d'enseignement systématique (enseignement de groupe - exercices individuels — évaluation critériée) utilisée quotidiennement et permettant d'évaluer, à partir de mini-tests, l'apprentissage des élèves et d'identifier les objectifs pédagogiques poursuivis par l'enseignant — autrement dit, les objectifs sont déterminés en fonction du contenu de l'évaluation, et non l'inverse comme cela est coutume de le faire;

(b) évaluer les effets de mesures rééducatives appliquées dans la classe ordinaire par un orthopédagogue auprès des enfants qui obtiennent un score inférieur à $90 \%$ lors de l'évaluation quotidienne;

(c) évaluer les effets de l'accessibilité d'une aire d'enrichissement en tant qu'activité renforçante chez les enfants qui obtiennent $90 \%$ ou plus à l'évaluation quotidienne (cette composante est une application du principe de Premack et les activités d'enrichissement sont choisies en fonction de la théorie des répertoires de la personnalité de Staats);

(d) évaluer l'efficacité de techniques de gestion de classe qui s'appuient sur les principes de l'analyse appliquée du comportement dans un contexte où les services de psychologie sont intégrés directement dans la salle de classe auprès de l'enseignant (cette 
formule correspond au niveau 2 - aide professionnelle offerte à l'enseignant dans la classe - du système en cascade proposé au cours des années 70 par Reynolds - voir rapport COPEX (1976) - afin de favoriser l'intégration des élèves en difficulté);

(e) évaluer les effets de l'autonotation quotidienne des résultats de l'élève sur un graphique comme le graphique d'enseignement de précision ou autres graphiques;

(f) évaluer les effets, auprès des parents, d'un rapport hebdomadaire relatif aux succès et aux progrès de leur enfant (seules les informations relatives à l'amélioration des comportements sociaux de l'enfant ou à son progrès scolaire sont transmises aux parents).

La structure de Primes permettait à un enseignant d'intégrer l'une ou l'autre des composantes du modèle, et ce, pour une, deux, trois ou quatre étapes de l'année scolaire et pour la lecture, l'écriture ou les mathématiques. L'idée ici est de respecter les choix des enseignants, favorisant ainsi une meilleure appropriation de la démarche.

De 1977 à 1978, le programme a été pré-expérimenté à l'école Marguerite-Bourgeois de la Commission scolaire Baldwin-Cartier, une école dont près de la moitié des élèves recevaient leur scolarisation dans des classes spéciales. De 1979 à 1984, le programme a été mis en place à l'école Jacques-Bizard de manière plus systématique, impliquant graduellement les enseignants de la première à la sixième année. Le curriculum scolaire a été divisé en 1222 objectifs quotidiens d'apprentissage en français et en 672 objectifs en mathématiques. Pour chaque objectif, un mini-test était construit par l'enseignant - en fait, l'objectif était construit à partir du contenu du mini-test. Au-delà des nombreux résultats recueillis au cours des années (voir Forget et al., 1988), il faut surtout retenir le fait que «pendant toutes les années du programme ", tous les enfants, sans exception et quels que soient leur difficulté, leur déficience ou leur handicap, ont pu poursuivre leur scolarité en classe ordinaire.

\section{Progrès}

Le Programme gradué avec renforcement pour les élèves suivis en tutorat (Progrès) a été développé par Marthe Langelier et Gilles Le Breton à la Commission scolaire des Moissons au cours des années 90 (Langelier \& Le Breton, 1992). Ce programme d'apprentissage de la lecture vise les élèves du primaire qui éprouvent des difficultés en français. 
En revanche, il peut s'adresser à l'ensemble des élèves en tant que matériel pédagogique accompagnateur. L'idée de départ des auteurs fut de concilier les principes du renforcement positif issus de la tradition en conditionnement opérant et du tutorat tels que développés par les programmes MÉLI et LIRE, et les orientations cognitives "globalistes » des programmes de lecture en vigueur au Québec à partir du milieu des années 80 . Le programme vise les élèves de la première à la troisième année. Le matériel se présente sous forme de fascicules appelés Tuto 1, Tuto 2 et Tuto 3. Les fascicules incluent un texte de lecture et une série de fiches de questions (Langelier \& Le Breton, 1992).

Les limites de Progrès sont nombreuses. D'une part, il manque un guide méthodologique expliquant la procédure de tutorat, la procédure d'inscription graphique des résultats des élèves et la procédure de renforcement. En fait, ce programme reprend essentiellement la démarche de $M E ́ L I$, faut-il le savoir. Ensuite, même si le programme a été expérimenté pendant deux ans à l'école Ste-Clotilde de la Commission scolaire des Moissons, les auteurs n'ont pas été en mesure de recueillir des données empiriques sur son efficacité, faute d'un financement à plus long terme.

\section{L'enseignement explicite de Boyer}

L'enseignement explicite s'est développé à partir de trois sources d'information. La première provient de l'observation des enseignants experts dans un domaine. La deuxième provient des études sur les enseignants qui réussissent auprès des enfants en difficulté d'apprentissage. Enfin, il s'inspire des résultats du projet Follow Throught déjà présenté.

Une séquence d'enseignement explicite se ramène généralement à trois étapes : le modelage, les exercices dirigés et les exercices autonomes. Dans un premier temps, l'enseignant identifie le type de raisonnement impliqué dans l'accomplissement de la tâche ou de la résolution du problème. Il rend explicite le «quoi », le «pourquoi », le «quand » et le « où faire ». Dans un deuxième temps, les élèves sont amenés à faire des exercices. L'activité permet à l'enseignant de vérifier le niveau de leur compréhension ou leur manière de faire. À cette étape, l'enseignant guide les élèves par des explications, des démonstrations et des rétroactions sur leurs essais. La plupart du temps, ces exercices se font en équipe. Dans un troisième temps, les exercices autonomes permettent aux élèves de réinvestir leurs acquisitions dans des activités individuelles. À cette étape, l'enseignant s'assure que les élèves maîtrisent les habiletés nécessaires à la réalisation de la tâche. 
L'enseignement explicite peut prendre trois modalités d'application. La première est l'enseignement dans le contexte d'une classe ordinaire où tous les élèves sont exposés aux diverses procédures. La deuxième est l'enseignement dans la classe ordinaire avec l'aide d'un enseignant spécialisé (comme un orthopédagogue) qui permet d'intensifier et d'individualiser les procédures pendant quelques heures par jour ou par semaine. La troisième est l'enseignement explicite intensif et individualisé. Ici, l'enseignement se fait en petits groupes à l'extérieur de la classe ordinaire et il revient souvent à un orthopédagogue d'assurer ce travail.

L'un des projets qui correspond à cette troisième modalité est le Programme DIR en lecture développé au début des années 90 par Christian Boyer. Il s'adresse à des élèves du primaire en difficulté d'apprentissage. Ce programme est intensif et se déroule sur une période de huit à dix semaines à raison de deux heures par jour, cinq jours par semaine. Les groupes sont constitués de 8 à 14 élèves. Au début de chaque séance, les objectifs sont précisés et l'enseignant explique en détail le raisonnement cognitif nécessaire à la réalisation de la tâche. Le rythme de présentation du matériel et des activités est très rapide. Ainsi, contrairement à la pédagogie traditionnelle ou aux différentes modalités inspirées du socioconstructivisme, l'enseignant ne suit pas le rythme des élèves. Il impose plutôt un rythme rapide afin de susciter continuellement leur attention. En enseignement explicite, comme dans tous les modèles de pédagogie comportementale, la motivation est considérée comme un facteur incontournable de l'apprentissage. Ici, la motivation provient de trois sources : le matériel pédagogique qui doit correspondre aux intérêts des enfants et qui doit faciliter le développement de leur motivation, les interactions avec l'enseignant qui doit constituer une source de renforcement social et des stimuli de renforcement matériel obtenus par l'intermédiaire de techniques opérantes avec jetons, ceux-ci étant attribués non pour leur attention à la tâche ou leurs comportements sociaux, mais bien pour leur rendement (Forget, 1984).

Sur le plan des activités pédagogiques, l'enseignement explicite inclut trois principales stratégies. L'enfant doit d'abord apprendre à décoder les lettres et les mots (cette stratégie correspond à l'entrée grapho-phonétique, appelée depuis plusieurs années, et sous l'influence de la psychologie cognitive, la conscience ou la sensibilité phonologique). Ensuite, il doit faire de la surlecture en lisant et relisant les mots et les phrases du texte jusqu'à ce qu'il présente un certain débit prédéterminé 
et avec une exactitude d'au moins $80 \%$. Enfin, la lecture raisonnée (angl. reciprocal teaching) consiste pour l'enseignant à expliquer les différents types de raisonnement que l'élève doit faire (" qu'estce que je dois faire? »; « pourquoi dois-je le faire? »; « quand dois-je le faire? ») et les stratégies cognitives qu'il doit acquérir (raisonner en lisant, comprendre une question, faire une inférence, se dépanner lors d'un bris de compréhension). Boyer (2010) note que l'enseignement de la compréhension doit aussi inclure une série d'habiletés : formuler une question, formuler une réponse, s'auto-questionner, analyser une question, retenir l'information lue, faire une inférence, chercher une information explicite et chercher une information implicite.

L'enseignement explicite intensif de Boyer (2010) n'affirme d'aucune manière que la compréhension en lecture découle directement de l'habileté à décoder, tout comme d'ailleurs le décodage ne découle pas de la compréhension (cette dernière affirmation se retrouve souvent dans la littérature socioconstructiviste; pourtant elle n'est aucunement appuyée par la recherche empirique; elle semble relever, à certains égards, de la pensée magique). Les deux habiletés doivent être enseignées de manière explicite et les activités qui leur sont associées doivent faire l'objet d'exercices de manière intensive. Sur le plan théorique, une métaanalyse de Swanson (1999) montre que les modèles pédagogiques les plus efficaces auprès des enfants en difficulté d'apprentissage combinent l'enseignement direct et explicite du décodage et l'enseignement direct et explicite de stratégies cognitives de la compréhension, ce qui correspond, pour Boyer, à ce qu'est l'enseignement explicite.

De 1990 à 2000, plus de 700 élèves du Québec et de l'Ontario ont participé au programme de Boyer. Les participants proviennent surtout d'écoles primaires, mais aussi de certaines écoles secondaires en contexte de cheminement particulier. Les enfants proviennent aussi bien de milieux francophones, allophones, d’immersion française, d'écoles de quartiers modestes sur le plan socio-économique, urbains ou ruraux.

Boyer (2010) note que les effets du programme sont intéressants. Les élèves qui ont suivi le programme présentent des gains variés : 10 à $15 \%$ présentent une performance (mesurée par le débit de lecture, l'exactitude, la compréhension littérale, la compréhension d'inférences et l'acquisition de stratégies de recherche d'informations) supérieure au groupe témoin; 30 à $35 \%$ obtiennent une performance équivalente; 20 à $25 \%$ obtiennent une performance légèrement inférieure; 30 à $35 \%$ obtiennent une performance nettement supérieure. En outre, les acquisitions se maintiennent à long terme. 
La principale lacune du programme de Boyer est qu'il n'a pas fait l'objet de réplications réalisées par des chercheurs indépendants. Le travail reste à faire.

\section{Conclusion}

Comme l'affirment Péladeau et Legault (2000), n'importe quel modèle pédagogique qui revendique l'importance de la pratique intensive des habiletés visées par un programme, qui insiste sur la nécessité de présentations claires et sans ambiguïté du contenu pédagogique de la part de l'enseignant, l'évaluation fréquente du progrès de l'enfant par l'utilisation répétée de tests critériés et formatifs basés sur l'évaluation en fonction du curriculum (angl. curriculum-based assessment), l'utilisation de stratégies de renforcement positif, l'apprentissage d'habiletés du simple au complexe, la poursuite d'un haut niveau de maîtrise d'une habileté ou la recherche de la fluidité dans la performance, est voué trop souvent à l'opprobre social en étant taxé de pratique pédagogique désuète et traditionnelle. En fait, dès qu'une proposition pédagogique s'appuyant sur une hypothèse comportementale est proposée depuis les trois dernières décennies dans les pays francophones, plusieurs chercheurs et intervenants en éducation montent au créneau de l'inconfort cognitif et émotionnel, peut-être parce que subjugués par un idéalisme subjectif et un relativisme radical qui voient l'enfant comme le plus important constructeur (ou le seul, pour les plus radicaux) de sa propre connaissance. Sur le plan institutionnel, la présence de telles attitudes permet de poser une question : n'y a-t-il pas un danger de déresponsabilisation des institutions éducatives lorsqu'il s'agit d'évaluer l'efficience et l'efficacité des services offerts lorsque les élèves ne progressent pas - et ils semblent être un certain nombre à le faire, au regard du taux de décrochage scolaire - , s'ils sont les premiers responsables de leurs connaissances disciplinaires et de leurs compétences? Il y a ici une vision romantique et idéaliste de l'enfant et de l'apprentissage qui, pour certains, devrait se réaliser dans le bonheur, la joie et le jeu. Pourtant, peu d'athlètes, de musiciens, de romanciers, de chefs cuisiniers, de cardiologues ou d'agriculteurs vont affirmer que leurs années d'apprentissage furent vécues dans la joie et l'aisance. Pourquoi en serait-il autrement dans le cas de l'apprentissage de la lecture, de l'écriture, des mathématiques ou des sciences biologiques? Qui peut revendiquer, même chez ceux très doués sur le plan intellectuel, que l'apprentissage des notions de calcul différentiel et intégral peut se réaliser en l'absence de pratiques répétées, d'encouragements fréquents 
et d'un professeur compétent qui possède sur le bout de ses doigts sa matière? Le plus désolant est que les enfants les plus démunis sont ceux qui subissent les plus grands dommages de cette culture quelquefois jovialiste de l'apprentissage. Et comme le souligne une récente recherche de L'Archêveque et Forget (soumis), même les enfants talentueux semblent avoir des difficultés à profiter des bienfaits de la Réforme. Ainsi, dans cette étude, 187 élèves du primaire, candidats à des programmes d'éducation internationale dont la scolarité s'est entièrement déroulée à l'intérieur de la Réforme scolaire du Québec, obtiennent des scores inférieurs à un test de créativité (pensée divergente) par rapport aux scores de 187 élèves équivalents qui ont réalisé leur étude à l'école primaire avant l'implantation de la Réforme. Les résultats de cette étude sont importants parce qu'ils vont à l'encontre d'affirmations souvent avancée par les tenants du socioconstructivisme pédagogique selon lesquelles, pour les paraphraser, la Réforme pose sans doute des problèmes en regard des enfants en difficulté, mais s'avère bénéfique pour la majorité des tout-venant parce qu'elle favoriserait l'émergence de compétences transversales comme la créativité. En fait, il n'existe, à notre connaissance, aucune étude utilisant un protocole de recherche avec groupe témoin équivalant au projet Follow Through qui permet de soutenir les affirmations de ces chercheurs.

La critique des principes sous-jacents aux réformes d'orientation socioconstructiviste n'est cependant pas nouvelle. Ainsi, dès 1982, le linguiste français Jacque Capelovici réagissait à l'arrivée de ce type de pratique didactique. Citant certains extraits d'une lecture présentée lors d'une conférence de psychopédagogie comme «Produire une griffe de construction-transformation du savoir qui prévoit la multiplicité du processus ou degrés de construction, d'élaboration de la connaissance chez les sujets apprenants. Le sujet apprenant identifie l'objet, repère les relations répétables... pour intégrer finalement la notion dans le réseau des relations acquises. ", Capelovici ne put s'empêcher de parler d'un "Himalaya de cuistreries aux prétentions "pédagogiques" si ce n'est que les auteurs de ces invraisemblables élucubrations émargent (sic) au budget de l'éducation nationale... » (p. 209).

Et que dire des affirmations de certains pour qui une trop grande exercisation peut avoir des effets délétères sur l'ampleur de certaines aires du cerveau? Et que dire des affirmations de von Glasersfeld (2009) qui soutient que ses préférences épistémologiques et ses choix pédagogiques ne peuvent souffrir d'aucune justification, car ils seraient par essence indécidables? Autrement dit, il n'y aurait aucune 
justification empirique, rationnelle ou éthique aux choix pédagogiques du socioconstructivisme radical.

Nous voulons souligner ici deux problèmes posés par ces affirmations. D'une part, pourquoi privilégier la version radicale du socioconstructivisme telle que proposée par von Glasersfeld (l'un des consultants auprès du ministère de l'Éducation du Québec lors de l'implantation de la Réforme scolaire) plutôt que les propositions de Perrenoud (1997), celles de Meirieu (1993) ou le socioconstructivisme structuraliste plus récent de Dubidon (2011)? Sans doute que le premier est plus articulé sur le plan théorique, mais celui de Dubidon (2011) est certainement plus ludique, favorisant peut-être une meilleure appropriation du contenu par l'apprenant. Évidemment, la question est ouverte et seule une démarche empirique pourrait jeter un regard critique devant ces alternatives.

Nonobstant ces distinctions, la pédagogie socioconstructiviste devient, dans ce contexte radical, un monde parallèle au monde naturel, un monde néoberkelien et néoplatonicien qui ne peut concevoir d'autres alternatives que ses propres voies, celles du solipsisme intégral. Il s'agit bien d'un monde parallèle parce qu'il serait difficile pour un astrophysicien, un ingénieur civil ou un chirurgien contemporain de justifier son modèle théorique, ses propositions technologiques ou ses pratiques médicales en fonction de "choix indécidables». Depuis plusieurs années, la société contemporaine juge approprié de rendre responsables sur le plan éthique et déontologique les professionnels quant aux meilleurs moyens qu'ils doivent prendre pour répondre aux requêtes de leurs clients. D'ailleurs, les codes de déontologie des professionnels prévoient que l'un de leurs devoirs est de s'assurer que les moyens qu'ils utilisent ne causent pas de tort et qu'ils doivent exercer leur profession selon des principes scientifiques généralement admis. Pourquoi alors exiger des médecins, des psychologues, des ingénieurs, des avocats et, dans certains États, des enseignants euxmêmes, de respecter de telles contraintes éthiques et déontologiques et en même temps ne jamais s'intéresser à la responsabilité de ceux qui continuent à exiger des instances publiques d'utiliser des méthodes et des stratégies qui cherchent non seulement à éviter de s'appuyer sur des données probantes, mais qui affirment d'emblée qu'elles ne peuvent souffrir d'évaluations empiriques et scientifiques? On n'est pas loin ici des revendications de lacaniens français comme Miller (2005) qui, devant les méta-analyses démontrant coup sur coup l'efficacité des thérapies cognitivo-comportementales par rapport à la psychanalyse, 
affirment que la psyché et la souffrance humaine ne peuvent souffrir d'un regard empirique et de mesures ordinaires d'évaluation clinique.

Pour l'élève, apprendre à lire, à écrire et à résoudre des problèmes d'algèbre n'est pas un art, encore moins au tout début du processus d'acquisition. En revanche, cela peut le devenir une fois que l'habileté est maîtrisée et fluide, comme chez un maître. Manifestement, plusieurs tenants du socioconstructivisme radical semblent avoir bien peur des maîtres efficaces et des exigences élevées qu'ils défendent auprès de leurs élèves pour que ces derniers deviennent des personnes autonomes capables d'exploiter leurs compétences et d'explorer de manière pragmatique et créative leur environnement. Pour l'enseignant, ce phénomène nécessite non seulement des connaissances sur les processus fondamentaux d'apprentissage, mais aussi d'être exposé de manière objective à une science pédagogique et une technologie de l'enseignement qui existent depuis longtemps et qui ont la qualité de s'appuyer sur des données probantes, ou tout au moins de ne jamais reculer devant le test de la réalité, celui de la réussite probante et substantielle des élèves (Greer, 1994).

Les chercheurs, les auteurs et les praticiens qui critiquent depuis longtemps plusieurs principes sur lesquels s'appuient les réformes pédagogiques actuelles se font reprocher de ne proposer aucune alternative ou aucune solution pratique. Nous espérons que le présent texte, au-delà de ses prises de position épistémologiques fortes, illustre le fait qu'il existe une panoplie d'alternatives. La question est de savoir pourquoi elles ne sont pratiquement jamais enseignées dans les facultés, les départements d'éducation ou dans les programmes de psychologie de l'éducation. Watkins (1997) propose l'idée que ces alternatives remettent trop en question certaines manières de penser du milieu universitaire en éducation, de conseillers pédagogiques et de consultants d'éditeurs de matériel pédagogique qui, dans certains cas, ne font qu'un.

Dans certains domaines de la réadaptation et de la scolarisation comme la déficience intellectuelle ou les troubles du spectre de l'autisme, il revient à des organisations de parents d'avoir obligé les institutions publiques à remettre en question leur pratique en regard du concept de données probantes. Dans le domaine de l'éducation spécialisée, l'échec manifeste des réformes scolaires envers les enfants en difficulté fait encore trop souvent l'objet d'un déni. En 2010, un défenseur québécois de la Réforme proclamait à la radio que la situation était loin d'être tragique puisque les résultats des élèves qui terminaient leurs études secondaires 
et ceux des étudiants qui terminaient leurs études collégiales (Cégep) étaient supérieurs à ceux obtenus par des élèves d'autres états nordaméricains. Le drame ici est de passer sous silence le fait qu'un nombre trop important d'adolescents ne terminent pas leur école secondaire ou leur Cégep. C'est comme si on évaluait l'efficacité et l'efficience d'un programme d'intervention uniquement à partir des résultats de ceux qui réussissent à le terminer en oubliant qu'un pourcentage éhonté décroche en cours de parcours. Et comme Watkins (1997) le souligne, tant que le déni du problème existe, il n'y a pas grand-chose à faire, sinon de dénoncer, d'informer et de favoriser l'évaluation comparative et empirique des modèles d'enseignement privilégiés par les uns et par les autres.

\section{Références}

Adams, G., \& Engelmann, S. (1996). Research on direction instruction: 25 years beyond DISTAR. Seattle, WA: Educational Achievement Systems.

Akers, R.L. (1973). Deviant behavior: A social learning approach. Belmont, CA: Wadsworth.

Alberto, P. A., \& Troutman, A. C. (2003). Applied behavior analysis for teachers. Columbus, $\mathrm{OH}$ : Merrill.

Allard, G., Hivon, R., Leclerc, G., Mehu, H., Poulin, G., \& Riendeau, M. (1974). Connaissance des principaux modèles théoriques d'éducation. Québec, QC: Conseil supérieur de l'éducation.

Anderson, J. R., Reder, L. M., \& Simon, H. A. (1996). Situated learning and education. Educational Researcher, 25, 5-11.

Anderson, R. C. (1968). An analysis of a class of problem solving behavior. Final report. (ERIC Document Reproduction Service No. EDO20776)

Archambault, J. (1994). Le système d'émulation: un outil de motivation? Montréal, QC: Commission des écoles catholiques de Montréal.

Becker, W. C. (1977). Teaching reading and language to the disadvantaged - What we have learned from field research. Harvard Educational Review 47, 518-543.

Bégin, Y. (1978). L'individualisation de l'enseignement: pourquoi? Québec, QC: INRS-éducation.

Bégin, Y., \& Dussault, G. (1980). La pédagogie de la maîtrise ou la redécouverte du bon enseignant. Vie pédagogique, 10, 4-6.

Bélanger, O. (1982). Modification du rendement et de la coopération en mathématiques par un programme d'entraînement incluant une technique de renforcement et l'enseignement de précision. La technologie du comportement, $6,1221-1243$.

Bereiter, C., \& Engelmann, S. (1966). Teaching disadvantaged children in the preschool. Englewood Cliffs, NJ: Prentice-Hall. 
Binder, C. V. (1993). Behavioral fluency: A new paradigm. Education Technology, Oct., 8-14.

Bissonnette, S., Richard, M., \& Gauthier, C. (2006). Comment enseigne-t-on dans les écoles efficaces? Québec, QC: Les Presses de l'Université Laval.

Bloom, B. S. (1968). Learning for mastery. Instruction and curriculum. Evaluation Comment, 1, 1-12.

Bondy, A., \& Frost, L. (1994). The Picture-exchange communication system. Focus on Autistic Behavior, 9, 1-19.

Boulet, A. (1999). Changements de paradigme en apprentissage. Apprentissage et socialisation, 19, 13-22.

Boyer, C. (2010). Le programme orthopédagogique DIR en lecture. Montréal, QC: Éditions de l'Apprentissage.

Brophy, J. (1983). Effective classroom management. The School Administrator, 40(7), 33-36.

Burgess, R. L., \& Bushell, D. G. (1969). Behavioral sociology. New York, NY: Columbia University Press.

Burk, F. L. (1908). A course of study in grammar and handbook to accompany the California state series. Sacramento, CA: W. W. Shannon.

Burton, R. (1978). Rapport de l'évaluation du système individualisé. Montréal, QC: INRS-Éducation.

Cadieux, A., \& Boudreault, P. (2003). Effets d'une intervention parentale en lecture sur la connaissance du nom et des sons des lettres et la sensibilité phonologique d'élèves à risque. Revue des Sciences de l'Éducation, 29, 545-564.

Cameron, J., \& Pierce, W. D. (2002). Reward and intrinsic motivation. New York, NY: Bergin \& Garvey Press.

Capelovici, J. (1992). Guide du français correct. Paris, France: L'Archipel.

Carnine, D. W., \& Silbert, J. (1979). Direct instruction readin.. Columbus, $\mathrm{OH}$ : Merrill.

Carnine, D. W., Silbert, J., \& Kameenu, E. J. (1997). Direct instruction reading. Upper Saddle River, NJ: Prentice Hall.

Commission des écoles catholiques de Montréal (1973). Guide d’implantation du progrès continu. Progrès continu, 1-16.

Cooper, J. O., Heron, T. E., \& Heward, W. L. (1987). Applied behavior analysis. Upper Saddle River, NJ: Prentice-Hall.

COPEX (1976). Rapport du comité provincial de l'enfance inadaptée. L'éducation de l'enfance en difficulté d'adaptation et d'apprentissage au Québec. Québec, Canada: Ministère de l'Éducation.

Couffignal, L. (1963). La pédagogie cybernétique. Europe, 43, 433-434.

De Corte, E., Geerligs, T., Peters, J., Lagerweij, N., \& Vandenberghe, R. (1996). Les fondements de l'action didactique. Bruxelles: De Boeck, Belgique.

Déjean, A., Hottlet, C., \& Goulet, M. (1981). MELI. Méthode de lecture. Guide de l'enseignant. Montréal, QC: Division des Ressources, La commission scolaire des écoles catholiques de Montréal. 
Desrochers, M. N., \& Gentry, G. D. (2004). Effective use of computers in instruction. In D. J. Moran \& R. W. Malott (Eds.), Evidence-based educational methods (pp. 127-141). San Diego, CA: Elsevier Academic Press.

Dewey, J. (1916). Democracy and education: An introduction to the philosophy of education. New York, NY: Macmillan.

Dubidon, D. (2011). Pour un constructivisme structuraliste. Nunuche, 2, 3233.

Engelmann, S. (1969). Conceptual learning. San Rafael, CA: Dimensions Publishing.

Engelmann, S. (1980). Direct instruction. Englewood Cliffs, NJ: Educational Technology.

Epstein, R. (1991). Skinner, creativity, and the problem of spontaneous behavior. Psychological Science, 2, 362-370.

Evans, J. L. Homme, L. E., \& Glaser, R. (1962). The rule system for the construction of programmed verbal learning sequences. Journal of Educational Research, 55, 513-518.

Fontaine, O., Kulbertus, H., \& Etienne, A. M. (1996). Stress et cardiologie. Paris, France: Masson.

Forget, J. (1981). Primes: Programme de recherche sur l'implantation d'un modèle d'enseignement systématique. La Technologie du Comportement, 5, 99-142.

Forget, J. (1984). L'attention à la tâche et le rendement scolaire: des relations hiérarchisées. Apprentissage et Socialisation, 7, 100-117.

Forget, J. (2009). Dictionnaire des sciences du comportement. Montréal, QC: Laboratoire des sciences appliquées du comportement, Département de psychologie, Université du Québec à Montréal.

Forget, J., Otis, R., \& Leduc, A. (1988). Psychologie de l'apprentissage: théories et applications. Brossard, QC: Behaviora.

Gagné, R. M. (1965). The conditions of learning. New York: Holt, Rinehart and Winston.

Galperine, P. I. (1980). Essai sur la formation par étapes des actions et des concepts. In N. F. Talyzina (Éd.), De l'enseignement programmé à la programmation de la connaissance (pp. 167-183). Lille: Presses Universitaires de Lille, France.

Gambrill, E. D., \& Stein, T. J. (1978). Supervision in child welfare. Berkeley, CA: University of California.

Gardner, R., Sainato, D. M., Cooper, J. O., Heron, T. E., Heward, W. L., Eshleman, J. W., \& Grossi, T. A. (1994). Behavior analysis in education. Belmont, CA: Brooks/Cole.

Gauthier, C. (1994). Sans récompenses, sans punitions. Vie Pédagogique, 91, 9-11.

Gilbert, T .F. (1962). «Mathematics»: The technology of education. Journal of Mathematics, 1, 7-73. 
Giroux, N. (1979). Le modèle behavioral. La technologie du comportement, 3, 61-103.

Giroux, N., \& Forget, J. (1996). L'enseignement de précision: l'essor initial et les problèmes de maintien de la pratique. Science et Comportement, 24, 205-226.

Giroux, N., \& Forget, J. (2000). L’enseignement de précision: définition, rétrospective historique et validation d'une procédure graphique modifiée. Scientia Paedagogica Experimentalis, 37, 69-106.

Greer, R. D. (1994). A systems analysis of the behavior of schooling. Journal of Behavioral Education, 4, 255-264.

Greer, R. D., \& Keohane, D. D. (2004). A real science and technology of education. In D. J. Moran \& R. W. Malott (Eds.), Evidence-based educational methods (pp. 23-43). San Diego, CA: Elsevier Academic Press.

Gropper, G. L. (1974). Instructional strategies. Englewood Cliffs, NJ: Educational Technology Publications.

Harris, M. (1979). Cultural materialism. New York, NY: Simon \& Schuster.

Homme, L. (1970). How to use contingency contracting in the classroom. Champaing, IL: Research Press.

Hottlet, C. (1981). MELI ou la lecture reconsidérée. Apprentissage et Socialisation, 4, 26-40.

Hottlet, C., \& Déjean, A. (1980). Méthode expérimentale. Lecture individualisée. Rapport d'expérimentation. Montréal, QC: Division des Ressources, La commission scolaire des écoles catholiques de Montréal.

Ingvarsson, E. T., \& Handey, G. (2006). An evaluation of computer based programmed instruction for promoting teacher's greetings of parents by name. Journal of Applied Behavior, 39, 203-214.

Johnson, K., \& Street, J. (2004). The Morningside model of generative instrument. In D. J. Moran \& R. W. Malott (Eds.), Evidence-based educational methods (pp. 247-265). Cambridge, MA: Cambridge Center for Behavioral Studies.

Journault, L. (1982). Entraînement à la lecture d'élèves de maternelle à partir d'une méthode individualisée de lecture (MELI). La technologie du comportement, 6, 61-76.

Kagel, J. H., Battalio, R. C., \& Green, L. (1995). Economic choice theory. An experimental analysis of animal behavior. Cambridge, UK: Cambridge University Press.

Keller, F. (1968). Good-Bye teacher... Journal of Applied Behavior Analysis, $1,79-89$.

Kuhn, T. (1972). La structure des révolutions scientifiques. Paris, France: Flammarion.

Kulik, J. A., Kulik, C. C., \& Cohen, P. A. (1979). A meta-analysis of outcome studies of Keller's personalized system of instruction. American Psychologist, 34, 307-318. 
Langelier, M., \& Le Breton, G. (1992). Progrès: Programme gradué avec renforcement pour les élèves suivis en tutorat. Montréal, QC: Les éditions La Pensée.

L'Archevêque, M., \& Forget, J. (2010). La créativité comme facteur prédicteur de la réussite scolaire en français chez des élèves de sixième année $d u$ primaire. Manuscrit soumis pour publication.

Leduc, A. (1989). L'histoire d'apprentissage d'une " enfant sauvage ». Brossard, QC: Éditions Behaviora.

Mager, R. F. (1962). Preparing instructional objectives. Belmont, CA: Fearon.

Magerotte, G., \& Willaye, É. (2010). Intervention comportementale clinique. Se former à l'A.B.A. Bruxelles, Belgique: de Boeck.

Markle, S. M., \& Tiemann, P. W. (1967). Programming is a process. [Film]. Chicago, IL:University of Illinois.

Marquis, D., \& Lavoie, L. (1998). Enseignement programmé. Enseignement modulaire. Sainte-Foy, QC: Presses de l'Université du Québec.

Martens, B. K., \& Meller, P. J. (1990). The application of behavioral principles to educational settings. In T. B. Gutkin \& C. R. Reynolds (Eds.), The handbook of school psychology (pp. 612-634). New York, NY: John Wiley.

Mayer, R. E. (1996). History of instructional psychology. In E. De Corte \& F. E. Weinert (Eds.), International encyclopedia of developmental and instructional psychology (pp. 29-33). Oxford, UK: Elsevier Science / Pergamon Press.

Mechner, F. (1967). Behavioral analysis and instructional sequencing. In P. C. Lange (Ed.), Programmed instruction (pp. 81-103). Chicago, IL: Rand McNally.

Meirieu, P. (1993). L'envers du tableau: quelle pédagogie pour quelle école? Paris, France: ESF.

Merrill, M. D. (1996). Computer-based design for computer-aided instruction. In T. Plomp \& D. P. Ely (Eds.), International encyclopedia of educational technology (pp. 149-152), Oxford, UK: Pergamon Press / Elsevier Science.

Merrill, P. (1978). Hierarchical and information processing task analysis. Journal of Instructional Development, 1, 35-40.

Miller, J.-A. (2005). Jacques-Alain Miller répond aux anti-Freud. Le Point, no 1723 , p. 80.

Montambeault, R., Archambault, J., Nadeau-Savoie, N., \& Ouellet, G. (1979). Fiches de lecture. Programme L.I.R.E. Vol. 1 à 4. Montréal, QC: Commission des écoles catholiques de Montréal.

Montambeault, R., Archambault, J., \& Ouellet, G. (1979). Guide méthodologique du programme L.I.R.E. Montréal, QC: Commission des écoles catholiques de Montréal.

Moran, D. J., \& Malott, R. W. (2004). Evidence-based educational methods. San Diego, CA: Elsevier/Academic. 
Ninness, H. A. C., McCuller, G., \& Ozenne, L. (2000). School and behavioral psychology. Norwell, MA: Kluwer Academic.

Overall, K. L. (1997). Clinical behavioural medicine for small animals. St. Louis, MO: Mosby.

Pear, J., \& Kinsner, W. (1988). Computer aided personalized system of instruction. Machine-Mediated Learning, 2, 213-237.

Péladeau, N., Forget, J., \& Gagné, F. (2010). Le rôle du transfert des apprentissages dans l'acquisition des habiletés simples et complexes. In M. Crahay \& M. Dutrévis (Eds), Psychologie des apprentissages scolaires (pp. 47-63). Bruxelles: de Boeck.

Péladeau, N., \& Legault, A. (2000). Qui a peur de l'enseignement direct? In N. Giroux \& J. Forget (Eds.), Pour un nouveau départ assuré en lecture, écriture, mathématiques et autres apprentissages personnels et sociaux: guide pédagogique destiné aux enseignants en difficulté (pp. 120-133). Montréal, QC: Les conseillers en enseignement de précision.

Pennypacker, H. S., Koenig, C. H., \& Lindsley, O. R. (1972). Handbook of the standard behavior chart. Gainesville, FL: H.S. Pennypacker.

Perrenoud, P. (1997). Construire des compétences à l'école. Paris: Éditions sociales françaises.

Pressey, S. L. (1926). A simple apparatus which gives tests and scores and teaches. School and Society, 33, 373-376.

Pryor, K. (1984). Don't shoot the dog. New York, NY: Simon and Schuster. Quintin, M. P. (1982). Auto-notation et auto-évaluation: incidences sur le rendement en lecture. La technologie du comportement, 6, 177-193.

Rhine, W. R. (1981). Making schools more effective: New directions from Follow Through. NewYork, NY: Academic Press.

Rosenshine, B. V., Stevens, R. (1986). Teaching functions. In M. C. Wittrock (Ed.), Handbook of research on teaching (pp. 376-391). New York, NY: Macmillan.

Scandura, J. (1966). Prior learning, presentation order, and prerequisite practice in problem solving. Journal of Experimental Education, 34, 1218.

Scandura, J. (1973). Structural learning. New York, NY: Gordon \& Breach.

Schestakow, A. W. (1968). L'enseignement programmé et les machines à enseigner en U.R.S.S. Paris, France: Dunod.

Skinner, B. F. (1968). The technology of teaching. Englewood Cliffs, NJ: Prentice-Hall.

Skinner, B. F. (1957). Verbal behavior. Englewood Cliffs, NJ: PrenticeHall.

Staats, A. (1986). Béhaviorisme social. Brossard, QC: Éditions Behaviora.

Stebbins, L., St. Pierre, R., Proper, E., Anderson, R., \& Cerva, T. (1977). Education as experimentation. Cambridge, MA: Abt Associates. 
Swanson, H. L. (1999). Reading research for students with LD: A metaanalysis of intervention outcomes. Journal of Learning Disabilities, 32, 504-532.

Talyzina, N. F. (1980). De l'enseignement programmé à la programmation de la connaissance. Lille, France: Presses Universitaires de Lille.

Tardif, J. (1998). Intégrer les nouvelles technologies de l'information : quel cadre pédagogique? Paris, France: ESF.

Tardif, J., \& Presseau, A. (1998). Intégrer les nouvelles technologies de l'information. Paris, France : ESF.

Thorndike, E. L. (1912). Educational psychology. New York, NY: Teachers College, Columbia University.

Ulman, J. (1979). A critique of "Skinnerism: Materialism minus the dialectic". Behaviorists for Social Action Journal, 1, 1-8.

Vargas, J. S. (2009). Behavior analysis for effective teaching. New York, NY: Routledge.

Vargas, E. A., \& Vargas, J. S. (1992). Programmed instruction and teaching machines. In R. P. West \& L. A. Hamerlynck (Eds.), Designs for excellence in education (pp. 33-69). Longmont, CO: Sopris West.

von Glasersfeld, E. (2009). Key works in radical constructivism. Rotterdam, The Netherlands: Sense Publishers.

Vygotsky, L. S. (1986). Thought and language. Cambridge, MA: MIT Press.

Watkins, C. L. (1997). Project Follow Through. Cambridge, MA: Cambridge Center for Behavioral Studies.

Watson, J. B. (1914). Behavior. New York, NY: Holt.

Watson, J. B. (1919). Psychology from the standpoint of a behaviourist. Philadelphia, PA: Lippincott.

West, R. P., \& Hamerlynck, L. A. (1992). Designs for excellence in education. Longmont, CO: Sopris West.

Wolery, M., Bailey, D. B, \& Sugai, G. M. (1988). Effective teaching. Principles and procedures of applied behavior analysis with exceptional students. Boston, MA: Allyn and Bacon.

Wolpe, J. (1958). Psychotherapy by reciprocal inhibition. Stanford, CA: 
Stanford University Press.

\section{Correspondance :}

Jacques Forget

Laboratoire des sciences appliquées du comportement

Département de psychologie

Université du Québec à Montréal,

C.P. 8888, Succ. Centre-ville

Montréal, Québec, Canada H3C 3P8

forget.jacques@uqam.ca

\section{Résumé}

La pédagogie comportementale est apparue au début du $20^{\mathrm{e}}$ siècle avec les travaux d'Edward Lee Thorndike, Frederick Lister Burk et Sidney Pressey. Il faudra cependant attendre l'ouvrage de B.F. Skinner, The technology of teaching, publié en 1968, pour que cette conception de l'enseignement trouve une place appréciable parmi les modèles pédagogiques disponibles dans les écoles. Combiné à l'émergence de nombreuses techniques d'analyse de tâche, d'orientation comportementale ou cognitive, l'enseignement programmé et ses variantes se sont développés de manière importante au cours des années 60 et 70. La technologie de l'instruction est alors devenue un domaine de recherche et une orientation pédagogique légitimes. Dix ans plus tard, l'arrivée d'une conception cognitive « globaliste» de l'apprentissage a rapidement dominé la pensée didactique. Quinze ans plus tard, cette orientation s'est transformée en didactique socioconstructivisme, la version pédagogique du postmodernisme qui se situe aux antipodes de la tradition empirique et du doute rationaliste en attribuant, dans sa version radicale, comme celle de von Glasersfeld (2009), la même valeur épistémologique à toutes les formes de connaissances, qu'elles soient scientifiques, intuitives ou mythiques. L'approche soutient aussi que toutes les compétences d'un enfant, qu'elles soient disciplinaires ou transversales, ne peuvent provenir que de sa propre construction subjective. Mais au-delà de ces transformations, l'enseignement programmé a masqué l'apport de nombreux travaux de recherche qui furent développés dans le contexte de l'éducation spécialisée et de projets d'intégration scolaire et qui donnèrent lieu au développement de 
modèles et de méthodes d'enseignement s'appuyant sur la psychologie comportementale. Le présent article vise à faire un survol de ces principaux modèles. À la fin de ce panorama, quelques expériences réalisées au Canada français au cours des 50 dernières années sont brièvement présentées.

Mots-clés : enseignement direct; enseignement personnalisé; enseignement de précision; enseignement systématique; enseignement explicite.

\section{$* * *$}

\section{Abstract}

Behavioral models of schooling have existed since the work of Edward Lee Thorndike, Frederick Lister Burk and Sidney Pressey. The behavioral approach took animportantstep forward with the book byB.F.Skinner,The technology of teaching (1968) on programmed instruction. For laypeople and professional practitioners, the behavioral approach to education was exclusively associated with this type of technology. However, in the mid 60s and 70s, direct instruction, personalized instruction, and precision teaching represented some of the most important contributions of the behavioral approach to schooling. Since then, other models like $C A B A S^{\circledast}$ or Morningside model of generative instruction have been proposed. The purpose of this paper is threefold. First, it describes the characteristics of these models and their contributions to the field of special education. Second, it discusses some studies in behavioral education which have been attempted in some French schools in Canada. The third purpose is to challenge the validity of the socioconstructivist school reforms that have been established in Québec and other countries over the past 15 years. Compared with behavioral models of schooling, those reforms have never presented empirical-evidence to support their claims or their efficiency with disabled children.

Keywords: behavioral models of schooling; special education; projects in French schools; school reforms; socioconstructivism. 